\section{Leucemia linfóide aguda: uma doença curável}

\section{Acute lymphoblastic leukemia: a curable disease}

Francisco Pedrosa 1

Mecneide Lins 2

1-2 Serviço de Oncologia Pediátrica. Instituto Materno Infantil de Pernambuco, IMIP. Rua dos Coelhos, 300. Boa Vista. Recife, Pernambuco, Brasil. CEP: 05.070-550 e Centro de Hematologia e Oncologia Pediátrica

\begin{abstract}
Acute lymphoblastic leukemia (ALL) is the most common type of cancer in children, accounting for one third of all malignant neoplasias affecting them. ALL incidences in children in the United States are of approximately 3,4 cases per 100.000 children under fifteen years old. Up to the middle of last century leukemia was universally considered a fatal disease. The development of therapeutic combinations through the use of various cytotoxic drugs with or without bone marrow transplantation has increased cure percentages of children with ALL in over $80 \%$. Annually around 1.500 children with ALL in the United States are being cured. Nevertheless, of the 75.000 new ALL cases annually diagnosed throughout the world, around $60.000(80 \%)$ have no access to the new treatments and are excluded from the cure process. The challenge of making ALL a curable disease is being met with the report of survival rates over $50 \%$ for children treated in countries with limited resources. In El Salvador, Bonilla has achieved increasing the survival rates in children with ALL from 5\% to 50\%. In Recife, Pedrosa accomplished bringing the $29 \%$ cure rate in the 80 's to the $75 \%$ current cure rate.
\end{abstract}

Key words Leukemia lymphocytic, Child, Drug therapy
Resumo Leucemia linfóide Aguda (LLA), é o tipo mais comum de câncer infantil, constituindo cerca de um terço de todas as neoplasias malignas da criança. A incidência de LLA em crianças nos Estados Unidos é aproximadamente de 3,4 casos por 100.000 crianças menores de 15 anos de idade. Até a metade do século passado, as leucemias eram consideras universalmene, uma doença fatal. O desenvolvimento de combinações terapêuticas, utilizando diversas drogas citotóxicas com ou sem transplante de medula óssea, tem aumentado o percentual de cura da criança portadora de Leucemia linfóide Aguda em mais de 80\%. Anualmente cerca de 1.500 crianças com LLA, nos Estados Unidos, estão sendo curadas. Contudo, dos 75.000 novos casos de LLA, que são diagnosticados anualmente em todo o mundo, cerca de 60.000 (80\%) não têm acesso a essas modernas formas de tratamento e são, portanto excluídos desse processo de cura. O desafio de tornar a LLA uma doença curável começa a ser vencido com o relato de taxas de sobrevida maior que $50 \%$ para crianças tratadas em países com recursos limitados. Em El salvador, Bonilla, conseguiu aumentar a taxa de sobrevida de crianças com LLA de 5\% para 50\%. No Recife, Pernambuco, Brasil, Pedrosa evoluiu de uma taxa de $29 \%$ nos anos 80 , para $75 \%$ na era atual.

Palavras-chave Leucemia linfocítica, Criança, Quimioterapia 
A Leucemia linfóide aguda (LLA) é o tipo mais comum de câncer infantil, constituindo cerca de um terço de todas as neoplasias malignas da criança. ${ }^{1}$

A incidência de LLA em crianças nos Estados Unidos é aproximadamente de 3,4 casos por 100.000 crianças menores de 15 anos de idade, com um pico maior de incidência ocorrendo entre três e quatro anos de idade. A LLA é mais comum em crianças brancas do que em negras $(1,8: 1)$ e em meninos do que em meninas $(1,2: 1)$.

O tratamento da LLA é prolongado, variando de dois a três anos. Embora os esquemas terapêuticos possam mudar de centro para centro, os protocolos modernos invariavelmente são constituídos de cinco grandes fases: indução da remissão, intensificaçãoconsolidação, reindução, prevenção da leucemia no sistema nervoso central (SNC) e continuação ou manutenção da remissão.

$\mathrm{Na}$ indução geralmente são utilizadas três ou quatros drogas: corticóide, vincristine, L-asparaginase e daunoblastina. Teoricamente considera-se que a terapia da leucemia seria curativa se o tratamento precoce fosse suficiente para erradicar as células malígnas antes que elas se tornem resistentes às drogas. Esse conceito levou ao desenvolvimento de protocolos progressivamente mais intensivos com o aumento do número de drogas, especialmente para pacientes com alto risco de recaída. ${ }^{2}$

Os protocolos atuais apresentam percentuais de remissão completa de $98 \%$ a $99 \% .3$

A fase intensificação-consolidação é indicada para erradicar as células leucêmicas residuais, creditando-se a essa fase a melhora dos resultados. 4

A prevenção da recaída da leucemia no SNC é parte integral do tratamento curativo da LLA. Ela pode ser feita de maneiras diferentes, mas normalmente mediante o uso de quimioterapia intratecal e radioterapia do crânio. $\mathrm{O}$ conhecimento de que a radioterapia do crânio pode causar importante neurotoxicidade e ocasionalmente tumores cerebrais, especialmente em crianças mais jovens, 5 tem resultado na substituição dessa modalidade de terapia por doses adicionais de quimioterapia intratecal e uma quimioterapia sistêmica mais intensiva.

Embora a maioria das crianças com LLA apresentem altas taxas de sobrevida, com tratamento de manutenção com doses-padrão de 6-mercaptopurina e methotrexate, as LLAs de alto risco apresentam melhores resultados com tratamentos mais intensivos.

O século XX assistiu a um notável progresso não só em relação a um melhor conhecimento, mas também quanto ao tratamento do câncer infantil, principalmente as leucemias. ${ }^{6}$ Até a metade do século pas- sado, as leucemias eram consideradas, universalmente, uma doença fatal.

O desenvolvimento de combinações terapêuticas, utilizando diversas drogas citotóxicas com ou sem transplante de Stem-cell, tem aumentado o percentual de cura da criança portadora de Leucemia linfóide aguda em mais de $80 \% .7$ Essa acentuada melhora nos resultados tem produzido um aumento na população de sobreviventes. Anualmente cerca de 1.500 crianças com LLA, nos Estados Unidos, estão sendo curadas. É estimado que atualmente um em cada 1.000 adultos jovens, com menos de 20 anos de idade, seja um sobrevivente do câncer. As complicações tardias representam outra área de investigação e têm fornecido informações importantes para o planejamento do tratamento inicial no sentido de evitar tais complicações.

Contudo, dos 75.000 novos casos de LLA, que são diagnosticados anualmente em todo o mundo, cerca de 60.000 (80\%) não têm acesso a essas modernas formas de tratamento e são portanto excluídos desse processo de cura. Nosso maior desafio, neste século, é tornar acessível esse notável progresso a todas as crianças portadoras de LLA, independentemente do local onde elas residam.

\section{Progresso no tratamento}

Os agentes alquilantes foram os primeiros citotóxi$\cos$ a serem introduzidos no tratamento das leucemias, em 1943, devido à toxidade hematológica do gás mostarda, observada durante a I Guerra Mundial.

O primeiro agente efetivo no tratamento da LLA foi um antifolato, a aminopterina, baseado no conhecimento de que o ácido fólico era essencial para a hematopoese normal.

A história da cura da leucemia (Quadro 1) iniciase, portanto, na metade do século passado com o famoso trabalho do Dr. Sidney Farber8 (Figura 1), publicado no New England Journal of Medicine, em 1948, em que ele descreve uma sobrevida por mais de cinco anos em 50 crianças portadoras de Leucemia linfóide aguda tratadas com aminopterina. Infelizmente representava menos que $1 \%$ de todas as crianças tratadas.

Esse trabalho foi seguido pelo desenvolvimento de duas novas drogas efetivas no tratamento da LLA: os corticoesteróides, em 1950; e as antipurinas em 1953. Em 1961, foi demonstrado que a vincristine, um alcalóide da vinca, era capaz de produzir remissão da LLA. 9 


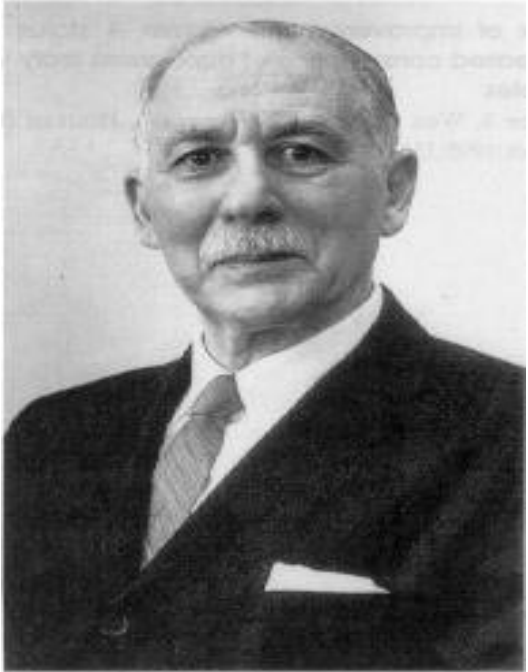

Sidney Farber (1903-1973).
"By 1952 Dr. Farber was able to describe a complete and persistent 5-year remission in a group of 50 children with leukemia. Sadly, they were but $1 \%$ of all the children treated. He believed strongly that continuous physiologic and psychological support must be given to the children being treated; he spent many hours with the parents of these children, as embodied in his emphasis on 'total care'." Graig J. Profiles in pediatrics II. J Pediatr 1996; 118 (1).
Quadro 1

Acontecimentos marcantes na cura da leucemia

\begin{tabular}{ll}
\hline 1865 & Trióxido de arsênico \\
1901 & Grupos sangüíneos \\
1902 & Radioterapia \\
1937 & Bancos de sangue \\
1943 & Agentes alquilantes \\
1948 & Antifolatos - Dona Farber \\
1950 & Corticoesteróides \\
1953 & Antipurinas \\
1954 & Transfusão de plaquetas \\
1955 & Grupos cooperativos \\
1961 & Vincristine \\
1962 & Protocolos com finalidades curativas - SJCRH \\
1971 & Sucesso do transplante de medula óssea
\end{tabular}

SJCRH = St. Jude Children's Research Hospital
No início dos anos 60, um regime com quatro drogas foi desenvolvido para tratamento da LLA, na tentativa de aumentar o percentual de remissão, somando-se os efeitos terapêuticos de cada droga, sem no entanto somar seus efeitos tóxicos. Foi reconhecido o papel importante do tratamento específico do sistema nervoso central na profilaxia de recaída meníngea, assim como a continuidade do tratamento por período de dois a três anos caracterizando a fase de manutenção do tratamento.

Nessa época, destaca-se o trabalho pioneiro do St. Jude Children's Research Hospital, em Memphis, nos Estados Unidos, com o desenvolvimento de protocolos terapêuticos com finalidade curativa designados de "Total therapy",10 com percentual de cura de $50 \%$ e seguimento por mais de 30 anos (Figura 2). 11 


\section{Figura 2}

Primeiro protocolo a proporcionar uma taxa de sobrevida de $50 \%$ em crianças portadoras de Leucemia linfóide aguda, com um seguimento de 32 anos demonstrando a durabilidade da cura.

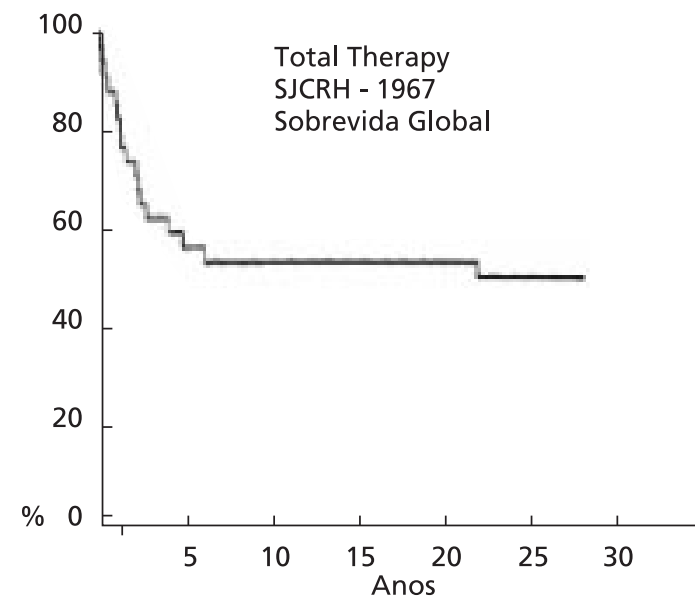

SJCRH= St. Jude Children' Research Hospital
Protocolos terapêuticos subseqüentes, nos últimos 25 anos, de várias instituições e grupos cooperativos, têm demonstrado o acentuado aumento do percentual de cura com índices atuais de $80 \%$ (Figura 3). ${ }^{12}$

O desafio de tornar a LLA uma doença curável começa a ser vencido com o relato de taxas de sobrevida maior que $50 \%$ para crianças tratadas em países com recursos limitados. Em El Salvador, Bonilla et al. 13 conseguiram aumentar a taxa de sobrevida de crianças com LLA de 5\% para 50\%.13 No Recife, Pedrosa (Comunicação pessoal) evoluiu de uma taxa de $29 \%$, nos anos 80, (Figura 4) para $75 \%$ na era atual (Figura 5).

\section{Figura 3}

Grande estudo multinstitucional confirmando a curabilidade da LLA em crianças e demonstrando a melhora da sobrevida nas últimas três décadas.
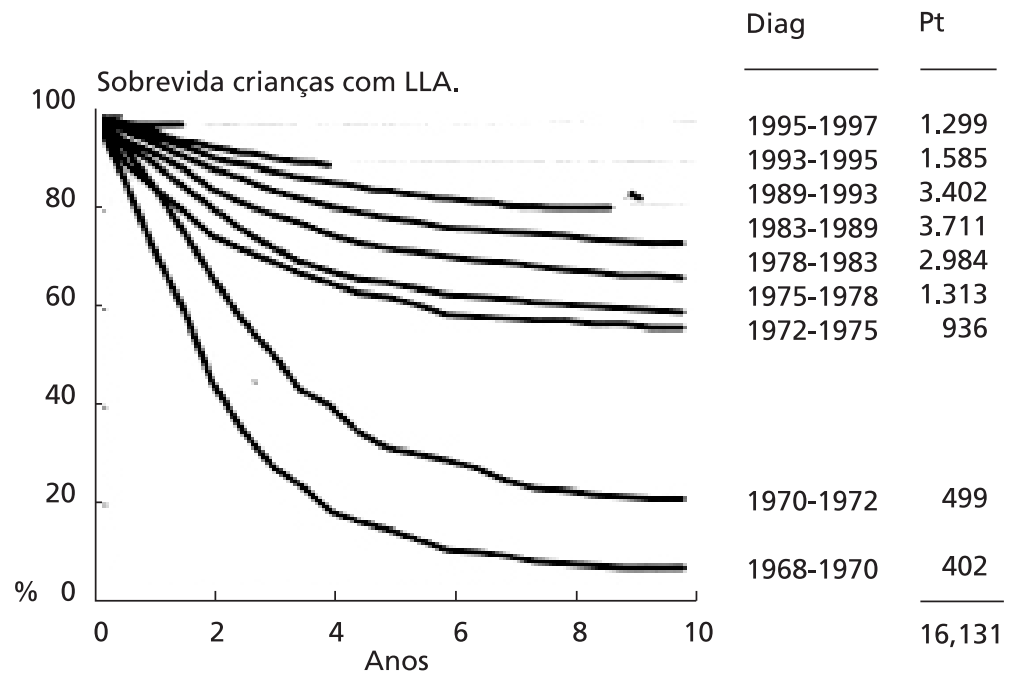

LLA = Leucemia Linfóide Aguda, Pt = Protocolo terapéutico Fonte: Cancer Children Group, 1968-1997.7 
Curva de sobrevida (Kaplan-Meier) de 91 crianças, portadoras de LLA de baixo e alto risco, tratadas, com o protocolo Total $X$ no Hospital Barão de Lucena. Recife, Pernambuco, Brasil, 1982 a 1987.

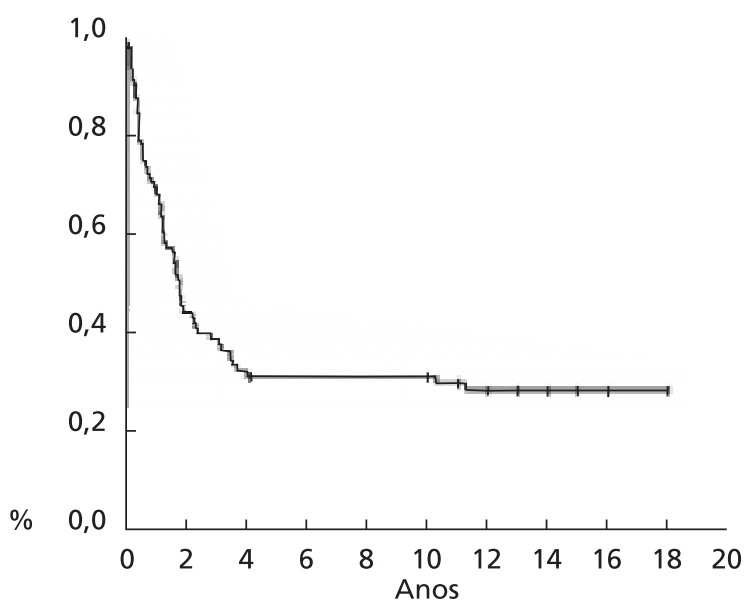

LLA = Leucemia Linfóide Aguda, PT = Protocolo terapéutico

\section{Figura 5}

Curva de sobrevida (Kaplan-Meier) de 147 crianças portadoras de LLA de baixo e alto risco, tratadas, com o protocolo Total XIIIB, no Instituto Materno Infantil de Pernambuco, IMIP. Recife, Pernambuco, Brasil, 1997 a 2001

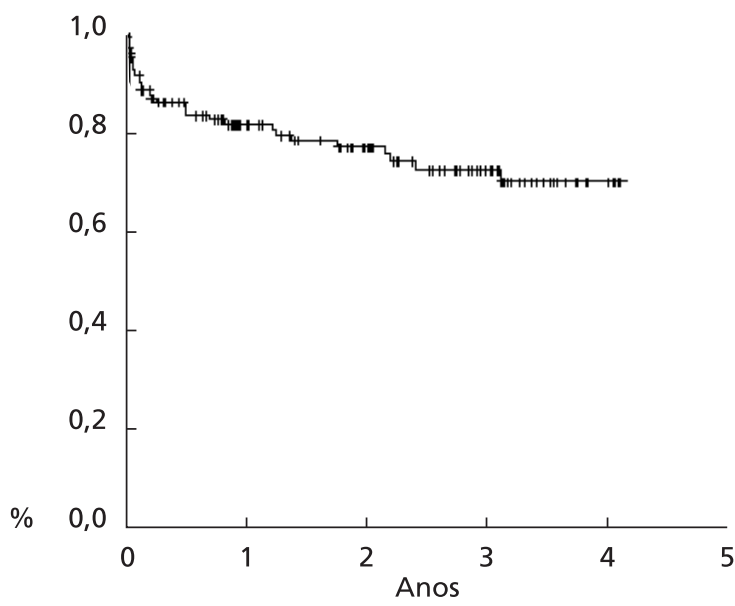

LLA = Leucemia Linfóide Aguda, PT = Protocolo terapéutico
A LLA evoluiu de uma doença mal definida e intratável na metade do século passado para uma doença que está entre as mais entendidas e as mais curáveis no início deste século.

Esse sucesso foi obtido graças não somente ao melhor conhecimento da doença, a introdução de novas drogas com protocolos terapêuticos adequados, mas sobretudo ao melhor tratamento de suporte.

$\mathrm{O}$ avanço do suporte hemoterápico, a utilização de fatores de crescimento de neutrófilos, o melhor controle da infecção e dos distúrbios metabólicos têm contribuído para esse sucesso.

No entanto, em nossa visão, o reconhecimento de que o câncer infantil deveria ser tratado por pediatras com formação em oncologia, e não por oncologista de adultos, foi o ponto de partida para essa dramática mudança.

A idéia do atendimento global, na qual a estratégia do tratamento é de atender a família como um todo e não somente a criança doente, oferecendo suporte psicológico e socioeconômico para que o tratamento possa ser realizado, com percentuais cada vez menores de abandono, faz parte desse conjunto de avanços que têm permitido ser a LLA uma doença cada vez mais curável.

Por fim é necessário destacar o papel importante das casas de apoio. Sem elas seria praticamente impossível tratar uma criança com câncer, sobretudo quando residente em área de recursos limitados. 


\section{Referências}

1. Gurney JG, Severson RK, Davis S, Robison LL. Incidence of cancer in children in the United States. Sex- race, and 1 -year age-specific rates by histologic type. Cancer 1995; 75: 2186-95.

2. Camitta B, Mahoney D, Leventhal B, Lauer SJ, Shuster JJ, Adair S, Civin C, Munoz G, Steuber P, Strother D. Intensive intravenous methotrexate and mercaptopurine treatment of higher-risk non-T, non-B acute lymphocytic leukemia: A Pediatric Oncology Group study. J Clin Oncol 1994; 12: 1383-9.

3. Pui CH. Acute lymphoblastic leukemia. Pediatr Clin North Am 1997; 44: 831-46.

4. Chessells JM, Bailey C, Richards SM. Intensification of treatment and survival in all children with lymphoblastic leukemia: results of UK Medical Research Council trial UKALL X. Medical Research Council Working Party on Childhood Leukemia [see comments]. Lancet 1995; 345 : 143-8.

5. Pui CH. Childhood leukemias. N Engl J Med 1995; 332: 1618-30.

6. Marina N. Long-term survivors of childhood cancer. The medical consequences of cure. Pediatr Clin North Am 1997; 44: 1021-42.

7. Brenner MK, Pinkel D. Cure of leukemia. Semin Hematol 1999; 36: 73-83.

8. Farber S, Diamond LK, Mercer LD et al. Temporary remissions in acute leukemia in children produced by folic acid antagonist, 4-aminopteroil-glutamic acid (Aminopterin). N Engl J Med 1948; 238: 787-92.
9. Karon M, Freireich EJ, Frei E 3rd, Taylor R, Wolman IJ, Djerassi I, Lee SL, Sawitsky A, Hananian J, Selawry O, James D Jr, George P, Patterson RB, Burgert O Jr, Haurani FI, Oberfield RA, Macy CT, Hoogstraten B, Blom J. The role of vincristine in the treatment of childhood acute leukemia. Clin Pharmacol Ther 1966; 7: 332-9.

10. Pinkel D. Five-year follow-up of "total therapy" of childhood lymphocytic leukemia. JAMA 1971; 216: 648-52.

11. Aur RJ, Simone J, Hustu HO, Walters T, Borella L, Pratt C, Pinkel D. Central nervous system therapy and combination chemotherapy of childhood lymphocytic leukemia. Blood 1971; 37: 272-81.

12. Pui CH, Evans WE. Acute lymphoblastic leukemia. N Engl J Med 1998; 339: 605-15.

13. Bonilla M, Moreno N, Marina N, de Reyes G, Shurtleff SA, Downing JR, Behm FG, Harrison PL, Ribeiro RC, Peña O, Crist WM, Antillon FG. Acute lymphoblastic leukemia in a developing country: preliminary results of a nonrandomized clinical trial in El Salvador. J. Pediatr Hematol Oncol 2000; 22: 495-501. 